\title{
La Transferencia del Aprendizaje en el Contexto de las Prácticas Laborales. La Formación Profesional en Cooperación con la Universidad y la Empresa
}

\author{
Programa: Recercaixa \\ Entidad que lo financia: La Caixa \\ Duración: 2 años (2013-2014)
}

\section{Resumen de la investigación:}

En el contexto socioeconómico actual, caracterizado por la economía del conocimiento, la competitividad y la globalización, nadie pone en duda el rol de la formación continua como elemento central para fortalecer la ventaja competitiva de las organizaciones. Sin embargo, se estima que el $10 \%$ del conocimiento, habilidades y actitudes desarrolladas en programas de formación relacionada con el puesto de trabajo son puestas en efectivamente práctica en el contexto laboral. Los programas formativos orientados al trabajo y el empleo, en muchas ocasiones, suelen ser puestos en tela de juicio de acuerdo a los bajos resultados obtenidos en general.

Esta situación plantea numerosos cuestionamientos desde el punto de vista de la gestión pedagógico-didáctico, y otros también, pero, sobre todo, desvela la cuestión de la transferencia del aprendizaje como una problemática a la que, los diseñadores, desarrolladores y formadores, debemos responder. Muchas son las dimensiones que intervienen en el proceso de transferencia del aprendizaje, pero, ¿qué podemos hacer para mejorar, facilitar y potenciar la transferencia de lo aprendido al puesto de trabajo? ¿Qué aspectos del diseño formativo favorecen el aprendizaje de nuevas habilidades y conocimientos susceptibles de ser transferidas al puesto de trabajo? ¿Cómo se puede mejorar la implementación de un programa formativo vinculado al trabajo? Si la transferencia del aprendizaje al puesto de trabajo es comprendida como un proceso recurrente de construcción de significados en los márgenes convergentes entre el contexto formativo y el contexto laboral, ¿es posible identificar elementos que favorezcan la transferencia del aprendizaje al puesto de trabajo? En 
este sentido, el presente proyecto tiene como meta aportar conocimiento e información para la mejora de la cooperación y sinergias existentes entre la Formación Profesional, la Universidad y la Empresa a través de la exploración e identificación de elementos claves del proceso formativo que tienen impacto en el proceso de transferencia del aprendizaje al puesto de trabajo.

\section{Objetivos:}

1. Identificar factores y condiciones de la relación entre 'diseño formativo de las prácticas - puesto de trabajo - participante' que favorecen o inhiben el proceso de transferencia del aprendizaje al puesto de trabajo.

- Explorar, analizar y formular indicadores objetivos que permitan la identificación de rasgos y características de la intervención didácticopedagógica que promuevan y favorezcan el aprendizaje de conocimientos y habilidades aplicables al puesto de trabajo.

- Explorar, analizar y formular indicadores objetivos que permitan la identificación de características de la naturaleza del trabajo y la organización que favorezcan o inhiban el proceso de transferencia de conocimientos y habilidades aplicables al trabajo.

- Identificar características personales de los participantes de la formación, tales como la motivación para aprender y desarrollo personal y profesional, que influyen el proceso de transferencia del aprendizaje al puesto de trabajo.

2. Elaborar una propuesta de mejora a través del diseño de un modelo de prácticas en el marco de la Formación Profesional en colaboración con la Universidad y la Empresa que recoja los indicadores mencionados, sus relaciones e interacciones.

- Describir y explicar las relaciones e interacciones entre los diferentes elementos del proceso de transferencia del aprendizaje e identificar posibles intervenciones para su mejora.

- Proponer líneas de trabajo para la mejora de la formación y su adecuación a las necesidades del puesto de trabajo y de los participantes.

- Diseñar y proponer un instrumento para la evaluación de la transferencia del aprendizaje en contextos de formación en y para el empleo.

3. Promover espacios de encuentros (físicos y virtuales) entre instituciones proveedoras de formación profesional para el empleo con el objetivo de analizar, discutir y mejorar la formación. 


\section{Miembros del equipo de investigación:}

Investigador Principal: Manel Fandos (Universitat Rovira i Virgili)

Otros miembros:

Dr. Ángel Pío González Soto (Universitat Rovira i Virgili)

Dr. José Tejada Fernández (Universitat Autònoma de Barcelona)

Dr. Jose Miguel Jiménez González (Universitat Rovira i Virgili)

Dr. Antonio Navio Gámez (Universitat Autònoma de Barcelona)

Dra. Carmen Ruíz Bueno (Universitat Autònoma de Barcelona)

Dra. Ana Inés Renta Davids (Universitat Rovira i Virgili)

Dr. Òscar Mas Torelló (Universitat Autònoma de Barcelona)

Dr. Aitor Gómez González (Universitat Rovira i Virgili)

Dra. Carme Garcia Yeste (Universitat Rovira i Virgili)

Dr. Òscar Flores Alarcia (Universitat de Lleida)

Joan Cabré Olivé (Universitat Rovira i Virgili) 